\section{BUTTERFLIES OF THE REGIONAL RESEARCH LABORATORY CAMPUS, JORHAT, ASSAM}

\author{
Mantu Bhuyan ${ }^{1}$, Pranab R. Bhattacharyya ${ }^{1,2}$ and \\ P.B. Kanjilal ${ }^{1}$
}

${ }^{1}$ Division of Plant Sciences \& Ecology, Regional Research Laboratory, Jorhat, Assam 785006, India

Email: ${ }^{2}$ pranabramb@yahoo.com (Corresponding author)

India possesses 1501 species of butterflies (Kunte et al., 1999) out of which northeastern India accounts for nearly a two-third (962 species) (Evans, 1932) of the species. Some reports of butterflies in certain areas of northeastern India have also been published by various workers (Mason \& Niceville, 1886; Doherty, 1889; Talbot, 1939, 1947; Wynter-Blyth, 1957; Saharia, 1967; Varshney \& Chanda, 1971). Recent surveys conducted in Kamrup district, Manas National Park, Nameri National Park and Dibru-Saikhowa Biosphere Reserve depict the status of a few butterfly species (Anon., 2002). However, the attempts still remain very fragmented.

Regional Research Laboratory (RRL) campus is situated $6 \mathrm{~km}$ westward from Jorhat town $\left(94^{0} 15^{\prime} \mathrm{E} \& 26^{\circ} 44^{\prime} \mathrm{N}\right)$. North to the RRL campus, the NH37 highway passes from Guwahati to Jorhat town. The campus occupies an area of $136.4 \mathrm{ac}$. During the establishment of the institute, $70 \%$ of the area was cleared for construction. However, in time, the gaps were filled with various endemic as well as exotic plant species. Towards the east of the main gate, an area of about $0.8 \mathrm{ac}$ has completely been converted to woodland with various strata of vegetation beneath the big canopies. Inside the campus, two gardens with medicinal plants of northeastern India were setup. Apart from these two gardens, one ecological park NEEP (North East Ecological Park) having various species of endemic plants of the region is present. The vegetation of the campus can be classified as tree patch plantation, avenue plantation, cultivable land and swampy land. The forest type is a mixture of evergreen, deciduous, grassland, hedge land and aquatic land. Among the plant species Eucalyptus sp., Albizia procera, Mesua ferea, Mimusops elengi, Terminalia belerica, Terminalia arjuna, Acularia agallocha, Aegle marmelos, Aristolochia indica, Paederia foetida etc. are dominant. The campus area has more than 150 species of various medicinal and aromatic plants. Detailed account of floristic diversity of RRL campus is not yet completed. However, a few plant species have been identified (Table 1). Several species of plants have already been identified as larval host plant of butterflies (Table 2) and detailed biology of few species of butterflies were also studied (Bhuyan et al., 2003a,b).

The butterflies were surveyed during the period 2001-2002. Adult butterflies were collected by net and preserved accordingly. The eggs and larvae observed during surveys were collected and reared in their respective host plants in the laboratory up to adult emergence. Accordingly, the adult butterflies were identified and preserved in the laboratory for future reference. During the study period a total of 70 species of butterflies belonging to 45 genera were recorded. Maximum numbers of species were observed in the family Nymphalidae (40) followed by Papilionidae (12), Pieridae (10), Lycaenidae (5) and Hesperiidae (3). The butterflies were identified and verified following Wynter-Blyth (1957), Evans (1932), Haribal (1992) and Kunte (2000). Butterflies collected during the period are presented in Table 3.

\section{REFERENCES}

Anonymous (2002). Butterflies and moths diversity. In. State Biodiversity Action Plan, Assam. Assam Science Society, State Nodal Agency, National Biodiversity Strategy and Action Plan, India. pp.96106.

Bhuyan, M., P.R. Bhattacharyya and D.J. Kataki (2003a). Host utilization efficiency of the butterfly Yellow Pansy, Junonia hierta Fab. (Nymphalidae: Lepidoptera) on the plant Barleria cristata Linn. In. Biodiversity of Eastern Himalayan Protected Areas (Ed. P P Baruah), Department of Botany, Handique Girls' College, Guwahati, Assam, India, pp.171-174.

Bhuyan, M., P.R. Bhattacharyya and D.J. Kataki (2003b). Winter life history of the butterfly Cethosia biblis on its host plant Passiflora racemosa Linn. in Assam. The Indian Forester 129:5 (654-656).

Doherty, W. (1889). Notes on Assam Butterflies. Journal of the Bombay Natural History Society 58: 118-134.

Evans, W.H. (1932). The Identification of Indian Butterflies. The Bombay Natural History Society, Bombay, 455pp.

Haribal, M. (1992). The Butterflies of Sikkim Himalaya and their natural History. Sikkim Nature Conservation Foundation (SNCF), Sikkim, $217 \mathrm{pp}+60 \mathrm{p} 1$

Kunte, K. (2000). India - A lifescape: Butterflies of Peninsular India. Indian Academy of Science, Universities Press, Hyderabad, India, $254 \mathrm{pp}+31 \mathrm{pls}$.

Kunte, K., A. Joglekar, G. Utkarsh and P. Padmanabhan (1999). Patterns of butterfly, bird and tree diversity in the Western Ghats. Current Science 77(4): 577-586.

Mason, W. and D. Niceville (1986). List of butterflies. Journal of the Asiatic Society 55: 78-49.

Saharia, D. (1967). A collection of butterflies of Assam Agricultural College, Jorhat, Assam. Journal of the Assam Agriculture College 8790 .

Talbot, G. (1939). The Fauna of British India including Ceylon and Burma. Butterflies Vol. 1. Reprinted edition. Today and Tomorrow's Printers and Publishers, New Delhi, 600pp.

Talbot, G. (1947). The Fauna of British India including Ceylon and Burma. Butterflies Vol. I1. Reprinted edition. Today and Tomorrow's Printers and Publishers, New Delhi, 506pp.

Varshney, R.K. and S.K. Chanda (1971). Butterflies of NE India. Indian Museum Bulletin 6: 27-53.

Wynter-Blyth, M.A. (1957). Butterflies of the Indian Region. Bombay Natural History Society, Bombay, 523pp+72pls.

\section{ACKnOWledgement}

The authors are thankful to Dr. D.C. Goswami, Scientist G, RRL, Jorhat for his inspiration and support throughout the study period. 
Table 1. Dominant plant species of the RRL campus.

\begin{tabular}{lll}
\hline 1. Acacia auriculiformis & 22. Cymbopogon citrates & 42. Michelia champaka \\
2. Aegle marmelos & 23. Cymbopogon flexuosus & 43. Murraya koenigii \\
3. Ageratum conyzoides & 24. Cymbopogon martini & 44. Neolamarckia \\
4. Alastonia schlaris & 25. Cymbopogon & \multicolumn{1}{c}{ cadamba } \\
5. Albizzia lucida & \multicolumn{1}{c}{ winterianus } & 45. Nerium indicum \\
6. Albizzia procera & 26. Embelica officinalis & 46. Ocimum sanctum \\
7. Amaranthus spinosus & 27. Erythrina stricta & 47. Oldenladia diffusa \\
8. Annona sp. & 28. Euralyptus globulus & 48. Paederia foetida \\
9. Aquillaria agallocha & 29. Ficus hispida & 49. Persia bombycina \\
10. Aristolochia indica & 30. Gmelina arborea & 50. Polyalthia longifolia \\
11. Artocarpus & 31. Hibiscus rosa sinensis & 51. Psideum guajava \\
heterophyllus & 32. Litsea chinensis & 52. Ricinus communis \\
12. Bauhinia purpurea & 33. Litsea cubeba & 53. Salmalia malaberica \\
13. Bixa orellana & 34. Litsea glutinosa & 54. Sesbania grandifolia \\
14. Calotropis procera & 35. Magnolia grandiflora & 55. Shorea robusta \\
15. Carica papaya & 36. Mallatus albus & 56. Spondia mangifera \\
16. Cassia fistula & 37. Mangifera indica & 57. Terminalia arjuna \\
17. Citrus limon & 38. Maringa oleifera & 58. Terminalia belerica \\
18. Citrus aurantifolia & 39. Melastoma & 59. Zizyphus jujuba \\
19. Citrus grandis & \multicolumn{1}{c}{ malabathricum } & \\
20. Citrus sinensis & 40. Melia azadirachta & \\
21. Cocos nucifera & 41. Mesua ferrea &
\end{tabular}

Table 2. Butterfly larval host plant in the RRL campus.

\begin{tabular}{|c|c|c|}
\hline S.No. & Butterflies & Larval host plant \\
\hline 1. & Troides & Aristolochia indica \\
\hline 2. & Graphium sarpedon & Cinnamomum camphora, Litsea chinensis \\
\hline 3. & Graphium doson & $\begin{array}{l}\text { Cinnamomum sp., Magnolia grandiflora, } \\
\text { Michelia champaka, Polyalthia longifolia }\end{array}$ \\
\hline 4. & Graphium agamemnon & $\begin{array}{l}\text { Annona sp., Cinnamomum sp., } \\
\text { Michelia champaka, Polyalthia longifolia }\end{array}$ \\
\hline 5. & Papilio clytia & Cinnamomum sp., Litsea chinensis \\
\hline 6. & Papilio polytes & $\begin{array}{l}\text { Aegle marmelos, Citrus aurantifolia, C. grandis, } \\
\text { C. limon, C. sinensis, Murraya koenigii }\end{array}$ \\
\hline 7. & Papilio demoleus & Murraya koenigii, Citrus sp. \\
\hline 8. & Euremasp. & Cassia sp. \\
\hline 9. & Catapsilia pyranthe & Cassia fistula \\
\hline 10. & Pieris sp. & Brassica oleraceae \\
\hline 11. & Neptis sp. & Bombyx sp. \\
\hline 12. & Moduza procris & Neolamarckia cadamba \\
\hline 13. & Euthalia aconthea & Magnifera indica \\
\hline 14. & Ariadne sp. & Ricinus communis \\
\hline 15. & Danaus sp. & Calotropis procera, Nerium oleander \\
\hline 16. & Euploea sp. & Ficus sp., Nerium oleander \\
\hline 17. & Castalius rosimon & Ziziphus sp. \\
\hline 18. & Gangara thyrsis & Cocos nucifera, Ornamental palm \\
\hline
\end{tabular}

Table 3. List of butterflies of the Regional Research Laboratory, Jorhat campus.

\begin{tabular}{|c|c|c|c|}
\hline Common Name & Scientific Name & Common Name & Scientific Name \\
\hline \multicolumn{2}{|l|}{ Papilionidae } & Grey Count & Tanaecia lepidea Butler \\
\hline Common Bird Wing & Troides helena Linnaeus & Common Baron & Euthalia aconthea Cramer \\
\hline Golden Bird Wing & Troides aeacus C \& R Felder & Yellow Pansy & Junonia hierta Fabricus \\
\hline Common Mime & Papilio clytia f. dissimilis Linnaeus & Lemon Pansy & Junonia lemonias Linnaeus \\
\hline Great Mormon & Papilio memnon f. typical Linnaeus & Peacock Pansy & Junonia almana Linnaeus \\
\hline Tailed Red Breast & Papilio memnon f. alcanor Linnaeus & Grey Pansy & Junonia atlites Linnaeus \\
\hline Common Mormon & Papilio polytes Linnaeus & Blue Pansy & Junonia orithya Linnaeus \\
\hline Lime Butterfly & Papilio demoleus Linnaeus & Chocolate Pansy & Junonia iphita Cramer \\
\hline Yellow Swallowtail & Papilio machaon Moore & Blue Admiral & Kaniska canace Linnaeus \\
\hline Common Rose & Pachliopta aristolochiae Fabricius & Leopard Lacewing & Cethosia cyane Drury \\
\hline Red Helen & Papilio helenus Linnaeus & Red Lacewing & Cethosia biblis Fabricius \\
\hline Tailed Jaw & Graphium agamemnon Linnaeus & Tawny Rajah & Charaxes polyxena Felder \\
\hline Common Blue Bottle & Graphium sarpedon Linnaeus & Common Nawab & Polyura athamas Drury \\
\hline \multirow{2}{*}{\multicolumn{2}{|c|}{ Pieridae }} & Doub Branded Crow & Euploea sylvester Fabricius \\
\hline & & Common Indian Crow & Euploea core Cramer \\
\hline Great Orange Tip & Hebomoia glaucippe Linnaeus & Common Palm Fly & Elymnias hypermnestra Linnaeus \\
\hline Small Grass Yellow & Eurema brigitta Wallace & Spotted Palm Fly & Elymnias malelas (Hewitson) \\
\hline Chocolate Grass Yellow & Eurema laetae Moore & Common Evening Brown & Melanitis leda Linnaeus \\
\hline Common Emigrant & Catopsilia pomona Fabricius & Dark Evening Brown & Melanitis phidema Stoll \\
\hline Mottled Emigrant & Catopsilia pyranthe Linnaeus & Common Five Ring & Ypthima baldus Fabricius \\
\hline Red Base Jezebel & Delias aglaia (Linnaeus) & Common Brush Brown & Mycalesis perseus Fabricius \\
\hline Red Spot Jezebel & Delias descombesi (Boisduval) & Commander & Moduza procris Cramer \\
\hline Common Jezebel & Delias eucharis (Drury) & Common Castor & Ariadne merione Cramer \\
\hline Large Cabbage White & Pieris brassicae Gray & Angled Castor & Ariadne ariadne Linnaeus \\
\hline Indian Cabbage White & Pieris canidia Evans & Small Leopard & Phalanta alcippe Cramer \\
\hline \multicolumn{2}{|l|}{ Nymphalidae } & Common Leopard & Phalanta phalantha Drury \\
\hline Common Sergeant & Athyma perius (Linnaeus) & Nigger & Orsotrioena medus Fabricius \\
\hline Sullied Sailor & Neptis soma Moore & Common Dufler & Discophora sondiaca Westwood \\
\hline \multirow{3}{*}{$\begin{array}{l}\text { Common Sailor } \\
\text { Colour Sergeant }\end{array}$} & Neptis hylas Moore & \multicolumn{2}{|l|}{ Lycaenidae } \\
\hline & Parathyma nefte inara & Common Imperial & Cheritra freja (Fabricus) \\
\hline & (Doubleday \& Hewitson) & Silver Streak Aacacia Blue & Surendra todara De Niceville \\
\hline Orange Staff Sergeant & Parathyma cama (Moore) & Yam Fly & Loxura atymnus Fruhstorfer \\
\hline Yellow Jack Sailor & Lassipa viraja Moore & Centaur Oak Blue & Nilasera centaurus (Moore) \\
\hline $\begin{array}{l}\text { Common Map } \\
\text { Danaid Egg Fly }\end{array}$ & $\begin{array}{l}\text { Cyrestis thyodamas Boisduval } \\
\text { Hypolimnas misippus Linnaeus }\end{array}$ & Common Pierrot & Castalius rosimon Fabricus \\
\hline Clipper & Parthenos sylvia Cramer & Hesperiidae & \\
\hline Common Tiger & Danaus genutia Cramer & Chestnut Bob & Lambrix salsala (Moore) \\
\hline Plain Tiger & Danaus chrysippus Linnaeus & Dart & Cupitha purreea Moore \\
\hline Glassy Tiger & Parantica aglea Stoll & Common Dartlet & Oriens gola pseudolus (Mabille) \\
\hline
\end{tabular}

